\title{
Assessment of the frequency of routine removal of dental plaque prior to caries diagnosis by dentists in three cities in southern Brazil
}

\author{
Carlos Heitor Cunha Moreira ${ }^{(a)}$ \\ Julio Eduardo do Amaral \\ Zenkner(a) \\ Eduardo Machado(b) \\ Rachel de Oliveira Rocha ${ }^{(c)}$ \\ Luciano Casagrande (d) \\ Cassiano Kuchenbecker Rosing ${ }^{(e)}$
}

(a) MSc, Assistant Professor; (c) PhD, Assistant Professor - Department of Stomatology, School of Dentistry, Federal University of Santa Maria, Santa Maria, RS, Brazil.

(b) DDS, School of Dentistry, Federal University of Santa Maria, Santa Maria, RS, Brazil.

(d) PhD Student, Graduate Program in Dentistry, Federal University of Rio Grande do Sul, Porto Alegre, RS, Brazil.

(e) PhD, Adjunct Professor, Department of Conservative Dentistry, Federal University of Rio Grande do Sul, Porto Alegre, RS, Brazil.

\section{Corresponding author:}

Cassiano Kuchenbecker Rösing

Rua Dr. Valle, 433/701

Porto Alegre - RS - Brazil

CEP: 90560-010

E-mail: ckrosing@hotmail.com
Received for publication on Mar 02, 2008

Accepted for publication on Jul 14, 2008

\begin{abstract}
The aim of this study was to assess the frequency of routine use of dental prophylaxis prior to visual inspection, in order to diagnose caries, by dentists with different lapses of time after graduating time. One hundred and fifty one Brazilian dentists were interviewed in $3 \mathrm{Bra}-$ zilian cities to determine if they usually remove dental plaque prior to visual inspection for caries diagnosis. The dentists were stratified according to year of graduation. The association between the lapse of time after graduating and the practice of routinely removing dental plaque before clinical examination was tested using the chi-square test with a significance level of $5 \%$. Only $28.5 \%$ of the dentists reported that they usually remove dental plaque prior to clinical examination. The dentists who graduated in the last 15 years presented the lowest percentages of plaque removal prior to clinical examination $(15.1 \%)$, whereas the more experienced dentists reported that they perform prophylaxis more frequently. Of the professionals who graduated from 1960-1975, 23.9\% reported that they performed dental plaque removal prior to diagnosis, whereas the figure for those graduating from 1976-1990 was 46.2\%. Most of the dentists interviewed reported that they did not remove dental plaque prior to performing visual diagnosis of caries.
\end{abstract}

Descriptors: Dental plaque; Dental caries; Dental prophylaxis; Clinical practice variations. 


\section{Introduction}

Dental caries is classically described as an infectious and transmittable disease. Nevertheless, there is also evidence that it is not a classical infectious disease, and some studies in cariology literature classify dental caries differently, namely as a complex and multifactorial condition. Its marked behavioral characteristics associate it to other multifactorial pathoses such as cancer, cardiovascular diseases and diabetes. ${ }^{1}$ Its occurrence and rate of progression seem to be significantly decreasing. ${ }^{2,3,4}$ This fact renders non-cavitated lesions more meaningful to dental health care, especially because it is possible to arrest the disease. The changes observed in the prevalence of dental caries as well as the understanding of the mechanisms by which caries occurs and progresses have posed a new challenge to the diagnosis of dental caries. In the early stages of its clinical manifestation, restorative procedures are not indicated for the treatment of dental caries, which is desirable considering the goal of preserving the dental structure. The criteria that guide the diagnosis and clinical management of the disease have been gradually refined. The search for white spot lesions, allowing early intervention, has thus recently increased in importance. ${ }^{2}$ The same has occurred with the criteria for classifying lesions according to their activity. 5,6

White spot lesions are generally detectable by a very careful and detailed clinical examination, 5,7 and active lesions are often covered by dental biofilm. Thus, the removal of biofilm is a sine qua non condition for any visual examination of dental enamel intended to detect this kind of lesion. ${ }^{3,8}$ Traditional dentistry, focused on cavity preparation and on the need for restorative treatment, has turned into a practice engaged in diagnosing the healthdisease process, which includes the early detection of non-cavitated lesions and the interruption of the disease's natural history in order to preserve dental tissues, ${ }^{8,9}$ thus producing an improved effect on patient health. ${ }^{3}$ On the other hand, it is estimated that about half of the total caries experience will not be detected if the search for non-cavitated lesions is excluded from the diagnostic process. ${ }^{10}$ Thus, the removal of biofilm prior to visual inspection and the consequent early detection of white spot lesions are essential procedures for taking a modern and conservative approach to combating disease.

The present study assessed the frequency of plaque removal prior to visual examination of teeth with the objective of identifying caries lesions by dentists with different lapses of time after graduating.

\section{Material and Methods}

The data in the present study was collected through interviews conducted with dentists working in the cities of Santa Maria, Cruz Alta and Rosário do Sul, in the state of Rio Grande do Sul, Brazil. Professionals were identified through records supplied by the Regional Dental Council of the state of Rio Grande do Sul. In the city of Santa Maria, a randomly selected sample of dentists was drawn. After stratifying the dentists according to year of graduation, a probabilistic sample was obtained from all the dentists registered in the Regional Dental Council (registration is compulsory in Brazil). Contact was also attempted with all the professionals from the other two cities (census). In Santa Maria, if it wasn't possible to hold an interview after 3 attempts, the next consecutive dental professional registered in the Regional Dental Council was interviewed. The non-response rate was as follows: in Santa Maria, 27 dentists had moved from their registered addresses and 22 were not found after 3 attempts. In Cruz Alta, 14 had moved and 25 were not found after 3 attempts. In the city of Rosário do Sul, 1 dentist had retired, 1 had moved and 5 were not found after 3 attempts. None of the dentists contacted refused to participate in the survey. All the interviews were performed at the dental offices.

Before the interview, the study subjects were informed about the objectives of the study and the confidentiality adopted in relation to the information they would be giving. The dentists willing to participate in the study then signed an informed consent. The study design was revised and approved by the Institutional Review Board of the Federal University of Santa Maria, RS, Brazil.

A pilot study was conducted with 10 dentists to evaluate the questions to be administered in the 
interviews and the time needed to conduct the interview. The information obtained from these interviews was used to adjust the questions.

A total of 151 dentists were interviewed, 108 from Santa Maria, 28 from Cruz Alta, and 15 from Rosário do Sul. The year of graduation ranged from 1960 to 2005, and the median year was 1982. Most of the dentists were general practitioners, in that $52.8 \%$ of Santa Maria dentists, $75.9 \%$ of Cruz Alta dentists and $93.3 \%$ of Rosário do Sul dentists were general practitioners. The sampling procedures are described elsewhere in literature. ${ }^{11}$

The interview included several questions, and the closed question was whether the dentist usually removed dental plaque prior to clinical examination of the teeth. The answer could be "yes" or "no".

\section{Analysis of the results}

The dentists were stratified according to year of graduation. The association between the time after they graduated and the practice of routinely removing dental plaque before clinical examination was tested by the chi-square test. The level of significance was set at $5 \%$.

\section{Results}

Of the 151 dentists who were interviewed, 43 answered that they remove plaque prior to the clinical examination of teeth, corresponding to $28.5 \%$ of the valid answers, and 108 answered that they do not remove plaque prior to the clinical examination of teeth, corresponding to $71.5 \%$ of the sample.

When the dentists were stratified according to the length of time practicing their profession, those who graduated in the last 15 years presented the lowest frequency of plaque removal prior to clinical examination (18.6\%). This percentage was different from that of the two other strata, in that $55.8 \%$ of the dentists who graduated from 1976-1990, and $25.6 \%$ of those who graduated from 1960-1975 reported that they performed dental plaque removal prior to clinical examination (Table 1).

\section{Discussion}

The results of the present study showed that a high percentage of the dentists who were questioned do not remove dental plaque prior to clinical examination for caries diagnosis. Historically, caries diagnosis has focused on identifying cavitated lesions with the main purpose of establishing the patient's restorative treatment needs, considering that treatment has traditionally been solely related to dental structure damage. ${ }^{8}$ It is known that the clinical criteria used to identify caries lesions ranges broadly. ${ }^{8,12}$ However, the changes detected in the disease profile - reduction in occurrence and rate of progression of active lesions until cavitation ${ }^{2,3,4}$ - have been accompanied by a significant change in the diagnostic process. ${ }^{8,12}$ Greater attention has been given to the clinical detection of non-cavitated lesions that, in some cases, are the only clinical sign indicating the presence of disease and its activity. Moreover, the prevalence of non-cavitated lesions is higher than the prevalence of cavities, ${ }^{8,10}$ and the detection of these early signs of caries is not possible unless the teeth are clean and dry. Pitts, Fyffe ${ }^{13}$ (1988), in a clinical study of caries diagnosis, observed that when the detection of white spot lesions is included, the DMFT and DMFS are doubled, while the scores of individuals considered "caries free" decline to one fourth of the previously observed values. Furthermore, epidemiologic surveys have shown that white spot lesions are more prevalent than cavitated lesions in dentin. ${ }^{8,10}$ Considering this, it may be inferred that the actual conditions of the disease in the population have been underestimated, which consequently raises the question of a possible inadequate application of non-invasive therapeutic measures. ${ }^{7,14}$

Based on what was reported by the dentists interviewed, the present study has implications regarding the criteria used in the clinical identification

Table 1 - Reported routine removal of dental plaque previous to clinical examination stratified according to year of graduation.

\begin{tabular}{c|c|c|c}
\hline Year graduated & \multicolumn{1}{|c|}{ Yes } & No & $\mathrm{p}^{*}$ \\
\hline $1960-1975$ & $11(23.9 \%)$ & $35(76.1 \%)$ & 0.441 \\
\hline $1976-1990$ & $24(46.2 \%)$ & $28(53.8 \%)$ & 0.001 \\
\hline $1991-2005$ & $8(15.1 \%)$ & $45(84.9 \%)$ & 0.008 \\
\hline Total & $43(28.5 \%)$ & $108(71.5 \%)$ & \\
\hline
\end{tabular}


of caries lesions. The stratifying of the dentists into three groups according to three 15 -year periods of time after graduating allowed assessment of the association between the period of time elapsed since graduation of the professional and the adoption or non-adoption of the procedure of removing dental plaque prior to clinical examination. Although the diagnosis of caries is known to be a more complex process than the simple clinical search for lesions, ${ }^{15}$ identifying these lesions is the first step in this process, and is also essential for undertaking a more comprehensive diagnostic process. The detection of caries lesions allows an evaluation of the presence of disease activity and also of its past history, which has been quoted as the most important factor in caries risk prediction. ${ }^{16,17}$ The present survey indirectly evaluated the ability of 151 dentists who work in three cities of southern Brazil to identify non-cavitated lesions properly. Among the professionals with more work experience - those who graduated between 1960 and 1975 - only 11 (23.9\%) answered that they usually remove dental plaque before performing diagnosis. In contrast, $24(46.2 \%)$ of the professionals who graduated between 1976 and 1990 remove dental plaque before performing diagnosis, and only $8(15.1 \%)$ of the professionals who graduated between 1991 and 2005 do the procedure. It has thus been detected that only a sixth part of the dentists who graduated in the last 15 years and who participated in this study reported that they routinely remove dental plaque prior to clinical examination in search for caries lesions. These findings are apparently inconsistent with the great emphasis given in recent years to the inclusion of non-cavitated lesions in the diagnostic process. And it is also interesting to observe that the years in which the most significant breakthroughs occurred in carious process knowledge were precisely in the 80's (1980-1990). These findings indicate that even those professionals who graduated more recently do not keep abreast of current knowledge of the dental caries disease profile. In other words, they are not adopting a clinical practice consistent with the knowledge about the disease's diagnosis.

Today, the focus is on detecting caries lesions sooner and on adopting an early treatment ap- proach, thus leading to the preservation of dental structures. ${ }^{15,18}$ It should be borne in mind that the dental plaque removal procedure performed for the diagnosis of caries in a clinical environment does not remove biofilm completely. Working with extracted teeth, Reis et al. ${ }^{19}$ (2006) showed that the procedure of removing dental plaque in clinical conditions with rotary instruments was not enough to eliminate plaque from the enamel surface completely. Studies have shown, however, that the number of clinically detectable caries lesions can be significantly underestimated if prophylaxis is not performed prior to clinical diagnosis. As a consequence, considering the existence of undetected non-cavitated lesions, the number of individuals considered "caries free" may be greatly overestimated. ${ }^{13,20}$ In a study performed in Brazil in 2004, Assaf et al. ${ }^{14}$ (2004) found significant differences between the results of clinical examinations performed with the removal of plaque prior to examination and those of an epidemiologic survey performed following the WHO's recommendations ${ }^{21}$ by the same examiners and on the same subjects. This finding clearly demonstrates that non-cavitated lesions were widely underestimated in the epidemiologic survey. Additionally, none of the combinations used in the epidemiologic survey (using spatula, dental mirror, or dental mirror plus explorer probe, and with and without previous brushing and drying) were able to even closely equal the performance of the examination performed with a dental unit to detect non-cavitated lesions.

Considering the similarities between the clinical examination performed in a dental office without previous cleaning of the dental surfaces and one of the modalities of the epidemiologic examination tested by Assaf et al. ${ }^{14}$ (2004), it may be assumed that the professionals in that survey who said that they did not remove dental plaque prior to the examination are significantly underestimating the occurrence of non-cavitated lesions. The detection of any caries lesions in a non-cavitated stage is desirable because it allows non-invasive treatments to be administered..$^{18}$ In other words, a professional who does not consider non-cavitated lesions in his/her examination and focuses his/her therapeutic efforts solely on identifying cavities and restoring lost den- 
tal tissue may be assuming an individual who has only non-cavitated lesions to be a "caries free" individual. This approach is inconsistent with the current knowledge on the health-disease processes and prevents taking a more favorable approach to dealing with the disease in its initial and reversible stage.

\section{Conclusions}

Based on the results of the present study, it can

\section{References}

1. Fejerskov $\mathrm{O}$. Changing paradigms in concepts on dental caries: consequences for oral health care. Caries Res. 2004;38(3):18291.

2. Baelum V, Heidmann J, Nyvad B. Dental caries paradigms in diagnosis and diagnostic research. Eur J Oral Sci. 2006;114(4):263-77.

3. Fejerskov O, Kidd EAM. Dental Caries - The Disease and Its Clinical Management. London: Blackwell Munksgaard; 2003.

4. Pitts NB, Longbottom C. Preventive Care Advised (PCA)/ Operative Care Advised (OCA) - categorising caries by the management option. Community Dent Oral Epidemiol. 1995;23(1):55-9.

5. Ekstrand KR. Improving clinical visual detection - potential for caries clinical trials. J Dent Res. 2004;83(Spec No):C67C71.

6. Nyvad B, Fejerskov O. Assessing the stage of caries lesion activity on the basis of clinical and microbiological examination. Community Dent Oral Epidemiol. 1997;25(1):69-75.

7. Pitts NB. Diagnostic tools and measurements - impact on appropriate care. Community Dent Oral Epidemiol. 1997;25(1):24-35

8. Ismail AI. Clinical diagnosis of precavitated carious lesions. Community Dent Oral Epidemiol. 1997;25(1):13-23.

9. Fejerskov O. Concepts of dental caries and their consequences for understanding the disease. Community Dent Oral Epidemiol. 1997;25(1):5-12.

10. Amarante E, Raadal M, Espelid I. Impact of diagnostic criteria on the prevalence of dental caries in Norwegian children aged 5, 12 and 18 years. Community Dent Oral Epidemiol. 1998;26(2):87-94.

11. Moreira CHC, Zanatta FB, Antoniazzi R, Meneguetti PC, Rösing CK. Criteria adopted by dentists to indicate the ex- be concluded that a significant part of the dentists interviewed do not remove dental plaque prior to performing clinical examination, thus adversely affecting the diagnosis of incipient caries lesions. This finding raises the question of how effectively the knowledge provided by dental caries diagnosis research benefits the patients and populations served by dental professionals.

traction of periodontally involved teeth. J Appl Oral Sci. 2007;15(5):437-41.

12. Ismail AI. Visual and visuo-tactile detection of dental caries. J Dent Res. 2004;83(Spec No):C56-C66.

13. Pitts NB, Fyffe HE. The Effect of varying diagnostic thresholds upon clinical caries data for a low prevalence group. J Dent Res. 1988;67(3):592-6.

14. Assaf AV, Meneghim MC, Zanin L, Mialhe FL, Pereira AC, Ambrosano GMB. Assessment of different methods for diagnosing dental caries in epidemiological surveys. Community Dent Oral Epidemiol. 2004;32(6):418-25.

15. Nyvad B. Diagnosis versus Detection of Caries. Caries Res. 2004;38(3):192-8.

16. Alaluusua S. Salivary counts of mutans streptococci and lactobacilli and past caries experience in caries prediction. Caries Res. 1993;27(Suppl 1):68-71.

17. Disney JA, Graves RC, Stamm JW, Bohannan HM, Abernathy JR, Zack DD. The University of North Carolina caries risk assessment study: further developments in caries risk prediction. Community Dent Oral Epidemiol. 1992;20(2):64-75.

18. Angmar-Mansson B, Ten Bosch JJ. Advances in methods for diagnosing coronal caries - a review. Adv Dent Res. 1993;7(2):70-9.

19. Reis A, Mendes FM, Angnes V, Angnes G, Grande RHM, Loguercio AD. Performance of methods of occlusal caries detection in permanent teeth under clinical and laboratory conditions. J Dent. 2006;34(2):89-96.

20. Fyffe HE, Deery C, Nugent ZJ, Nuttall NM, Pitts NB. Effect of diagnostic threshold on the validity and reliability of epidemiological caries diagnosis using the Dundee Selectable Threshold Method for caries diagnosis (DSTM). Community Dent Oral Epidemiol. 2000;28(1):42-51.

21. World Health Organization. Guide to oral health epidemiological investigations. Geneva: WHO; 1979. 